\title{
Carbenes. Pincers, chelates, and abnormal binding modes*
}

\author{
Robert H. Crabtree \\ 225 Prospect St, New Haven, CT 06520-8107, USA
}

\begin{abstract}
Routes to pincer and chelate carbene complexes of $\mathrm{Pd}, \mathrm{Rh}$, and Ir include double geminal $\mathrm{CH}$ activation, metallation, and $\mathrm{CH}$ activation. Abnormal binding via imidazole $\mathrm{C} 5$ can occur, and ion pairing can strongly influence $\mathrm{C} 2$ vs. C5 binding. Prior ligand binding via pyridine $\mathrm{N}$ before metallation is not essential. Computational methods are used extensively, for example, to compare $\mathrm{N}$ vs. $\mathrm{C}$ binding in imidazole. The possibility of $\mathrm{C}$ binding by histidine in metalloenzymes is discussed.
\end{abstract}

\section{INTRODUCTION}

Fischer-type carbenes, [1] particularly those derived from imidazolium ions, [2] have attracted greatly increased attention since they have been shown to act as efficient spectator ligands in homogeneous catalysis [3]. Phosphines, $\mathrm{PR}_{3}$, the first major class of spectator ligands in organometallic homogeneous catalysis, are still by far the most widely used. Thanks to Tolman's parameters [4], they have predictable and tunable steric and electronic effects and they can be readily incorporated into chelates such as diphosphines and into asymmetric phosphines such as Kagan's diop [5] or Noyori's BINAP [6]. Fischer carbenes were discovered in the 1960s, but the $N$-heterocyclic carbenes (NHCs) subset of this group were only shown to be widely effective spectator ligands in catalysis in the mid-1990s, so the evolution of this field has been much retarded relative to the phosphine area. NHCs have since proved effective in a wide range of catalytic reactions, including hydrogenation, hydroformylation, Heck, Suzuki, Kumada, and Stille coupling, olefin metathesis, and $\mathrm{C}_{2} \mathrm{H}_{4} / \mathrm{CO}$ copolymerization. A number of significant reviews have appeared recently on the topic [3a,7].

In general, phosphines are nontrivial to synthesize but once they are obtained - and very many are now commercial - they can be readily bound to the metal, usually by simple mixing and reflux. NHC precursors-imidazolium salts - are often very easy to synthesize, but once obtained they can be more challenging than phosphines when it comes to binding them to the metal. Deprotonation at $\mathrm{C} 2$ with $n$-BuLi to give the free carbene is the classical procedure of Arduengo [8], but when an NHC is incorporated into a chelate or pincer ligand it is no longer clear where this deprotonation will occur and hence whether the complexation will proceed as intended or whether the very reactive intermediate carbene will survive to react with the metal.

Significant questions remain to be answered before NHCs can be considered as "tamed" for use in homogeneous catalysis and organometallic chemistry. What can go wrong in attempts to form a complex from the precursor salt and how do we combat these problems? What are the reactivity patterns of the NHC once bound and how do these impact on problems of catalyst deactivation? Can we slow deactivation pathways relative to productive catalytic steps? Can NHCs be usefully incorporated into pincer and chelate systems, and do these show slower deactivation? Is there any useful analogy between

\footnotetext{
*Plenary lecture presented at the $\mathrm{XX}^{\text {th }}$ International Conference on Organometallic Chemistry (ICOMC), Corfu, Greece, 7-12 July 2002. Other presentations are published in this issue, pp. 421-494.
} 
$\mathrm{PR}_{3}$ and NHCs, or are these better considered as quite separate ligands? Can we develop electronic parameters to help prediction of carbene properties? The work described in this review attempts to answer some of these questions.

\section{DISCUSSION}

\section{Acyclic carbenes}

In order to incorporate carbenes into pincer and chelate ligands, it seemed necessary to avoid the strong base deprotonation method of introducing the metal, since this could lead to deprotonation at undesired sites. Direct $\mathrm{C}-\mathrm{H}$ oxidative addition seemed a suitable method, so we looked at ortho-substituted pyridines that would form carbenes on cyclometallation. In initial work [9], we took an acyclic case. As shown in eq. 1, o-dimethylaminopyridine underwent a cyclometallation with $\left.\left[\mathrm{IrH}_{2} \text { (acetone }\right)_{2} \mathrm{~L}_{2}\right] \mathrm{BF}_{4}$ $\left(\mathrm{L}=\mathrm{PPh}_{3}\right)$ to give an equilibrating mixture of an alkyl hydride and a carbene dihydride. The reversible $\alpha$-elimination involved in this step is unusual. Since $\beta$-elimination is impossible in this system, we moved to the diethyl analog, but $\alpha$-elimination was still preferred, so even a single $\alpha$-nitrogen atom is sufficient to favor formation of a carbene over formation of an alkene. Other double $\mathrm{CH}$ activation routes to carbenes are known [10].

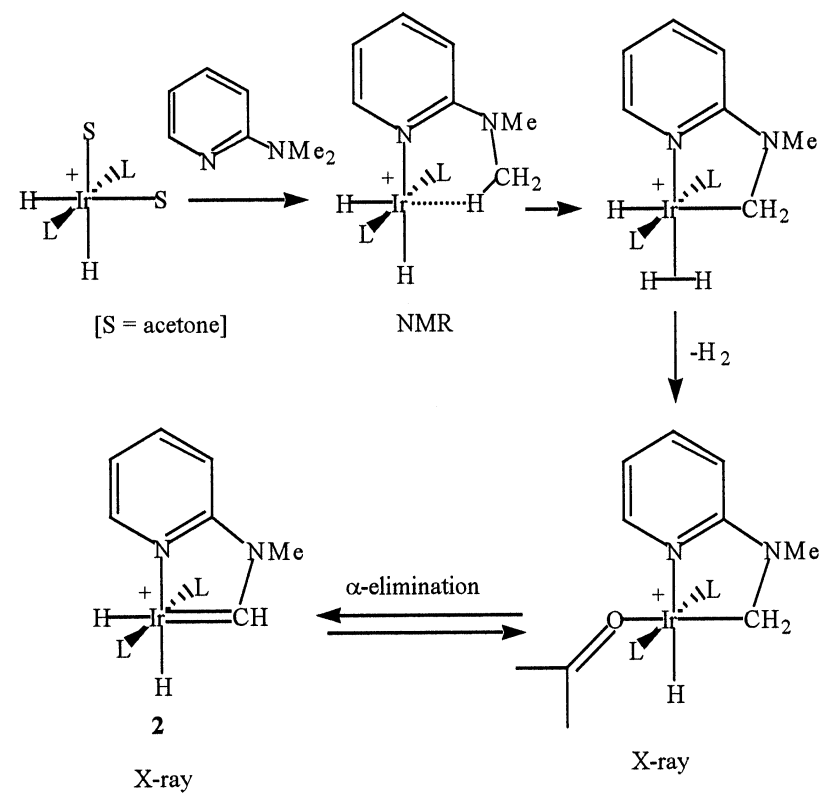

An agostic intermediate was detected by NMR spectroscopy. With the diethylamino analog, double geminal $\mathrm{CH}$ activation took place, even though a $\beta-\mathrm{H}$ is now potentially available for the elimination step.

\section{Palladium $\mathbf{N}$-heterocyclic pincer carbenes}

Moving to the cyclic case, we looked at metallation of imidazolium-substituted pyridines in collaboration with Eduardo Peris of Castellòn University [11]. With $\mathrm{Pd}(\mathrm{II})(\mathrm{OAc})_{2}$, metallation was possible with loss of $\mathrm{AcOH}$ as in eq. 2, where a CNC pincer complex of $\mathrm{Pd}(\mathrm{II})$ was formed and crystallographically characterized. This was a robust catalyst for the Heck reaction at $165^{\circ} \mathrm{C}$, showing the resistance of the complex to thermal decomposition; this is the case even in air. We feared $\operatorname{Pd}(0)$ might have been inadvertently formed and be the true catalyst so we used the $\operatorname{Hg}(0)$ test to eliminate this possibility. The pla- 
nar complexes had rather low solubility but using $\mathrm{R}=n$-Bu wingtip groups gave sufficient solubility for convenient study.

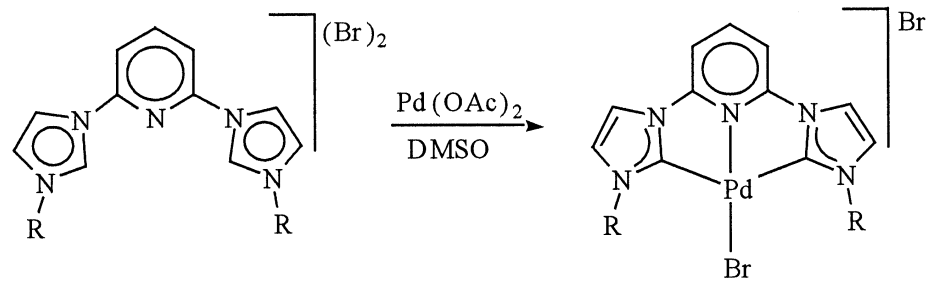

Introduction of a methylene linker proved possibly to give a pincer complex with a twisted structure determined crystallographically [12a]. The loss of planarity greatly improved the solubility. This is also a potentially chiral catalyst, but NMR measurements showed that the two chiral forms interconvert readily on warming and so we did not examine the possibilities for asymmetric catalysis. This would require blocking the atropisomerism with appropriate substitution at the benzyl linker unit. Both Cavell and Nolan have looked at related Pd(II) NHC complexes [13].

Since a CCC pincer was unknown and might rigidify the twisted structure sufficiently to slow or prevent interconversion of chiral forms, we looked at the appropriate bis-substituted arene precursor, $\mathbf{1}$. Lacking a metal-binding site, this failed to give a complex, and we were forced to look at alternate syntheses [12a]. The bromoaryl precursor shown in eq. 3 proved successful, but with a $\operatorname{Pd}(0)$ metallation reagent, not $\mathrm{Pd}(\mathrm{II})$. We presume the $\mathrm{Pd}(0)$ binds to the bromoaryl group by oxidative addition, followed by cyclometallation of the imidazole rings. $\mathrm{H}_{2}$ is presumably lost in this last step. The product was indeed much more rigid than the $\mathrm{CNC}$ pincer but was still fluxional enough to give coalescence at elevated temperature. Density functional theory (DFT) calculations by Eisenstein and coworkers [12b] give extremely good agreement with the experimental fluxionality barrier. All of these species were also catalytically active in the usual coupling reactions.<smiles></smiles>

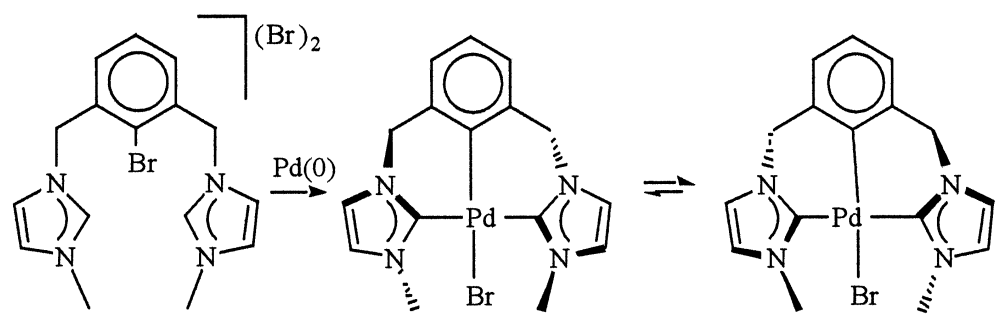

Having successfully employed $\mathrm{CBr}$ activation, we wondered if $\mathrm{CH}$ activation would be possible for similar types of imidazolium salts, as the literature suggested [13]. For this study [14] we chose the precursors $\mathbf{2} \mathbf{a}-\mathbf{b}$ as bromide salts. These proved to react with the same $\operatorname{Pd}(0)$ precursor, not to give a carbene hydride as we expected on the basis of precedent [13], but to give two types of bis-carbene, depending on the size of the R group; again, both were crystallographically characterized. This suggested 
that the intermediate hydride rapidly reacted with the imidazolium precursor to give the bis-carbene and, unexpectedly, $\mathrm{H}_{2}$. This could be a useful route in the future if it proves general.

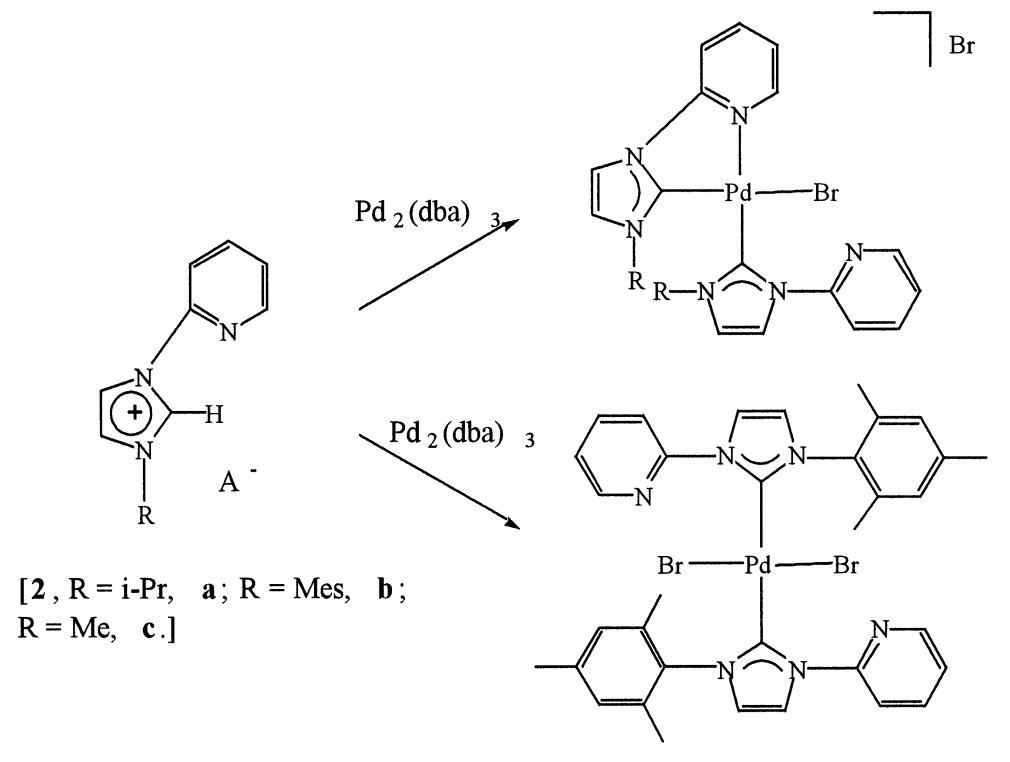

\section{Iridium NHCs: Right vs. wrong}

As a test of the hypothesis, based on eq. 4, that the acidic 2-position of an imidazolium salt would rapidly react with a metal hydride, we looked at the case of precursor $2 \mathbf{c}(\mathrm{R}=\mathrm{Me})$ as the $\mathrm{SbF}_{6}$ salt and $\mathrm{IrH}_{5} \mathrm{~L}_{2}\left(\mathrm{~L}=\mathrm{PPh}_{3}\right)$ [15]. Pyridine is known [16] to react with $\operatorname{IrH}_{5} \mathrm{~L}_{2}$ to give $\operatorname{Ir}\left(\right.$ py) $\mathrm{H}_{3} \mathrm{~L}_{2}$ (py = pyridine) so the initial product of the reaction with $2 \mathbf{c}$ should have the pyridine ring bound and the adjacent imidazolium ring in close proximity with an IrH group. This might be unobservable if it reacts further very fast. Indeed, as shown in eq. 5 , the product of the reaction $(\mathrm{R}=\mathrm{Me})$ is a carbene chelate, but to our great surprise, the metallation had taken place almost entirely at the C5 (4, "wrong") position rather than the usual C2 (3, "right"). This conclusion, confirmed by the spectral data and crystallography in collaboration with Jack Faller at Yale, means that metallation does not always give reaction at the presumed more reactive $\mathrm{C} 2$ position. In the presence of strong acid $\mathbf{4}$ can rearrange to $\mathbf{3}$, whatever anion was present, so we presume we see kinetic products in eq. 5. Chelation is not needed-imidazolium salts and $\mathrm{C}_{6} \mathrm{H}_{5} \mathrm{~N}$ give similar products.

Theoretical work (ONIOM, B3PW91/UFF) was carried out in collaboration with Eric Clot and Odile Eisenstein [17] of the University of Montpellier-2 by modeling the full complex, but with the R and $\mathrm{Ph}$ groups in the molecular mechanics part of the calculation. This suggested that in the absence of counterion, 3 should be significantly more stable than $\mathbf{4}$. When the anion is included in the model (in the quantum part) $\mathbf{3}$ and $\mathbf{4}$ now have similar energies because ion pairing with the counterion makes a significant energy contribution. This ion pairing leads to a preference for the wrong carbene because a stronger interaction occurs for the wrong carbene by hydrogen bonding of the acidic 2-C-H bond of 4 with the anion. Ion pairing with $\mathbf{3}$ is predicted to be less strong, since the 5-C-H bond of $\mathbf{3}$ is less acidic than the $2-\mathrm{C}-\mathrm{H}$ of 4 . Ion pairing therefore contributes to the stabilization of what is normally the less stable carbene in $\mathbf{4}$. Form $\mathbf{3}$ is not forbidden in this system, precursor $\mathbf{2 c}$ with a bromide counterion gave predominantly $\mathbf{3}$. Change of counterion allows us to bias the reaction almost entirely to $\mathbf{3}$ or $\mathbf{4}$ as desired. With a larger wingtip group, $i$-Pr instead of Me, the bromide salt gives $\mathbf{3}$, as before, but now moving to the $\mathrm{BF}_{4}$ salt gives $\mathbf{4}$ exclusively, again verified crystallographically. This work implies the counterion is not innocent in these systems. 

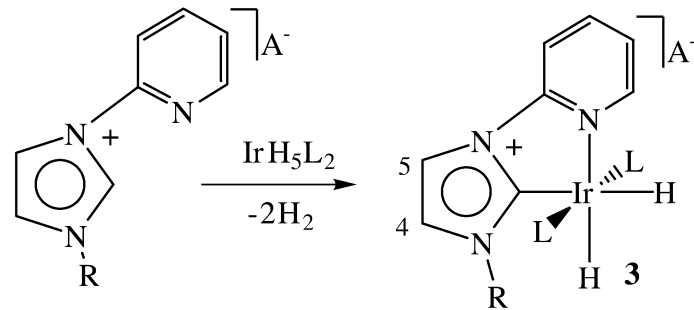

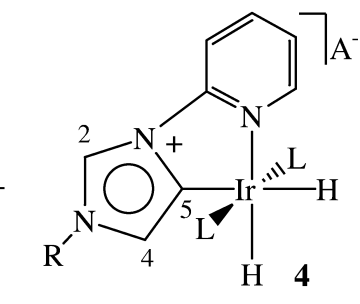

The new binding mode at $\mathrm{C} 5$ is characterized spectroscopically by the appearance of the acidic $\mathrm{C} 2$ proton at low field near $9 \delta$ in the proton NMR, shifted to ca. $11 \delta$ with bromide counterion. The chemical shift difference between the two imidazole ring protons is large-ca. $3 \delta$-as opposed to the smaller $\Delta \delta$ seen for the right carbene-ca. $1 \delta$. The two forms can also be distinguished in the carbon NMR because the right carbene has a resonance near $140 \delta$ as usual for NHCs, but the wrong-way carbene has its resonance at $170 \delta$ closer to that found for Fischer carbenes with one $\alpha-\mathrm{N}$ atom.

Free imidazolium salts are known to hydrogen bond strongly via the acidic 2-C-H [18]. We find that the 2-C-H proton NMR resonance of imidazolium salts is also very sensitive to counterion, showing a $2 \mathrm{ppm}$ low field shift on replacing $\mathrm{SbF}_{6}$ by $\mathrm{Br}$, suggesting that the ion pair structure seen in the crystal of $\mathbf{4}$ also applies to the solid state. The preferential stabilization of the transition state leading to 4 in the case of bromide is presumably responsible for the anion dependence of the $3 / 4$ ratio.

The generality of abnormal binding is still unclear and needs to be established in future work. Blocking the 4,5-imidazolium positions, for example by moving to the benzimidazolium analog, prevents abnormal binding, and a standard carbene complex is formed [19]. Blocking the C2 proton with a methyl group leads to exclusive abnormal carbene formation.

The mechanism remains obscure but we have evidence [19] that the formation of the abnormal carbene may be a result of a non-CH activation pathway. On stopping the reaction after 2 min instead of $2 \mathrm{~h}$, instead of the abnormal carbene, we find instead the hydrogenated carbene shown in eq. 6 . The ${ }^{13} \mathrm{C}$ NMR resonance of C5 now comes near $250 \mathrm{ppm}$, as in true Fischer carbenes. This species can be converted to the aromatic abnormal carbene on heating for $2 \mathrm{~h}$, so it seems to be an intermediate in the overall reaction. Using $\operatorname{IrD}_{5} \mathrm{~L}_{2}$ as metal complex leads to deuteration only at $\mathrm{C} 2$ and not $\mathrm{C} 4$. This suggests that the $\mathrm{H}$ originally at $\mathrm{C} 5$ migrates to $\mathrm{C} 4$ during the reaction of eq. 6 and is not transferred to the metal as would be the case if a $\mathrm{CH}$ activation pathway had been involved.

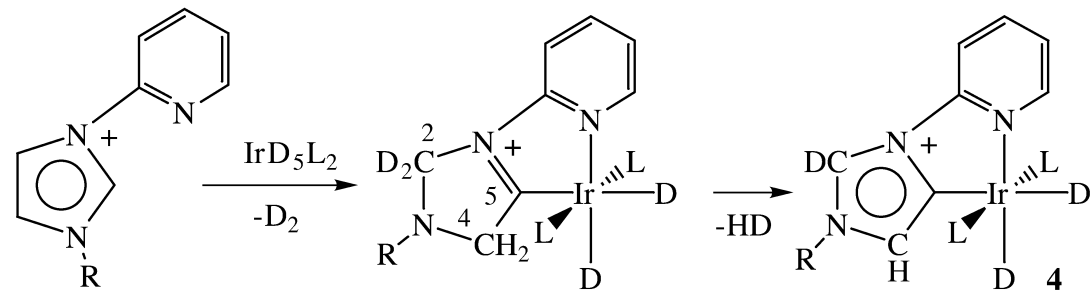

For the choice of anion to influence the outcome of the reaction of eq. 5 so strongly, the transition-state energies for the two pathways must differ substantially in their response to ion pairing. Tight ion pairs with a definite structure seem to exist in $\mathrm{CH}_{2} \mathrm{Cl}_{2}$ solution in these and related systems, as revealed in collaboration with Alceo Macchioni (Perugia), who uses a ${ }^{1} \mathrm{H},{ }^{19} \mathrm{~F}$ HOESY NMR technique that detects anion-cation contacts [20]. Theoretical work by Clot and Eisenstein [17,20a] suggests that the ion pair structure is determined by the shape of the electrostatic potential around the metal complex, excellent agreement between theoretical predictions and NMR data being obtained. I believe that minimal alteration of the ion pair structure is possible in the short time that elapses as the complex traverses the reaction barrier. If so, the anion effect on eq. 5 could plausibly arise from differences either in the absolute magnitude of the electrostatic field of the cation or in the way it changes in the transition-state (TS) region. Different TSs are expected to have different electrostatic potentials, and charge migration 
during a reaction is commonplace and is expected to be present in the TS. If one TS, say $\mathrm{TS}_{\text {right }}$, had a weak dipole or a change in dipole that caused decreased ion pair interaction, while the other TS, say $\mathrm{TS}_{\text {wrong }}$, had a large dipole or one that changed in such a way as to favor ion pairing, then anions like $\mathrm{Br}^{-}$capable of stronger ion pairing should favor the "wrong" product over the "right", as is observed.

The hydrogenation/dehydrogenation pathway of eq. 6 is unusual in that we have a redox active ligand, which can act as a source and sink of hydrogen atoms. If such ligand conversions can happen during the course of a catalytic reaction, the ligand could be an active participant rather than just a spectator. The electronic characteristics of the ligand could also be significantly modified by such an interconversion. At least in this system, right/wrong carbene interconversion has too high a barrier for it to take place rapidly enough to play a significant role in a typical catalytic reaction.

\section{N vs. C binding: Is C-bound histidine possible?}

The idea of alternate C-binding modes being possible in imidazolium ions is reminiscent of our suggestion [21] that a similar alternation, but between N- and C-binding modes, might obtain for imidazole itself and for histidine, the related amino acid. At the time, there was only one old report by Taube et al. [22] that $\mathrm{N}$ to $\mathrm{C}$ conversion is possible for an imidazole complex of $\mathrm{Ru}(\mathrm{II})$; more recently, Bergman et al. [23] reported an imidazole derivative that spontaneously metallates at $\mathrm{C}$ rather than binds at $\mathrm{N}$.

To test the idea of N/C binding equilibria, we turned to computational work (DFT, B3PW91) in collaboration with Gjergji Sini at the University of Cergy-Pontoise and Odile Eisenstein at the University of Montpellier-2 [24]. We predicted the thermodynamic preference for C2 vs. N1 binding in imidazole with a variety of metal fragments (i.e., the relative energies of $\mathbf{5 / 6}$ ). The results illustrate the general picture that although the imidazole is much stabler than its free carbene isomer, $\mathrm{N}$ - and C-binding modes to metals are not that different in energy. $\mathrm{N}$ binding tends to be preferred for first-row transition metals and $\mathrm{C}$ binding for second- and third-row metals, although this is a preliminary generalization that may be affected by future work.

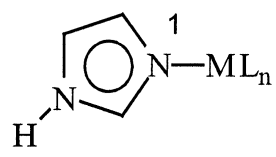

5

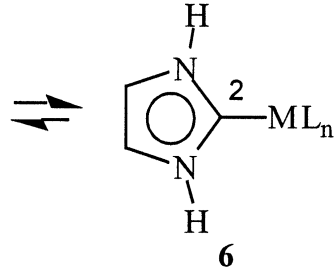

6

Because very many metalloenzymes employ histidine (His) as a metal-binding function, we considered the possibility that carbon binding might be possible in Nature. Because of the symmetry of His and the virtual identity of $\mathrm{C}$ and $\mathrm{N}$ in protein crystallographic data, a C-His would almost certainly have escaped detection. We are currently looking for anomalies or other indications for nonclassical C-His in proteins. The barrier for interconversion is likely to be quite high so a protein would probably have to contain suitable functionality to promote the interconversion. Herrmann has recently entertained similar views [3a].

\section{Tolman-style parameters for NHCs and related ligands}

One of the convenient features of working with phosphines is the availability of Tolman [4] steric and electronic parameters to guide the choice of system. We wanted to get some idea of how carbenes relate to phosphines in electronic effect. The steric comparison seemed more difficult to attack because of the fan-like shape of the carbene vs. the cone of $\mathrm{PR}_{3}$. Tolman's electronic parameters (TEPs) rely on 
the highest-energy IR $v(\mathrm{CO})$ stretching frequency for $\mathrm{LNi}(\mathrm{CO})_{3}$. While phosphines readily react with $\mathrm{Ni}(\mathrm{CO})_{4}$ to give the required species, the majority of interesting nonphosphine ligands, including NHCs, have not been found to give such complexes, at least so far. In our DFT (B3PW91) computational work, in collaboration with Perrin, Clot, and Eisenstein [25], we predicted the $v(\mathrm{CO})$ stretching frequency for $\mathrm{LNi}(\mathrm{CO})_{3}$, where $\mathrm{L}$ is obviously no longer restricted to cases where the complex-or even the ligand-is experimentally available. Initial work on cases where TEPs are known showed an extremely good correlation between prediction and experiment for $\mathrm{LNi}(\mathrm{CO})_{3}$, after a correction factor had been applied. Moving to the cases of unknown L, we were able to establish calculated electronic parameters (CEPs) for a variety of $\mathrm{L}$.

The general picture that emerges is that neutral $\mathrm{L}$ are found in a separate range of $v(\mathrm{CO})$ relative to anionic $\mathrm{X}^{-}$ligands, the latter being systematically much stronger donors. NHCs are found to be strongly donor relative to other neutral ligands. If they are regarded not as carbenes but as metallated imidazolium salts, they are anionic ligands modified by the presence of a positive charge on the ring, so on this model their strong donor power is not surprising.

An interesting feature of the results was the good correlation with available Lever parameters (LEPs). [26] The TEP and LEP sets of ligands have few common members so it had not been possible to interrelate these two electronic parameters. Now we see that both the TEP and LEP correlate well with the CEP, it seems likely that all three metrics are measuring the same fundamental property of the ligand. This was not at all obvious because the probe of the CEP and TEP, the $\mathrm{Ni}(\mathrm{CO})_{3}$ fragment, might have behaved quite differently from the probe for the LEP, which involves a redox potential implicating both $\mathrm{Ru}(\mathrm{II})$ and $\mathrm{Ru}(\mathrm{III})$.

\section{Chelating NHCs without other binding sites}

In the work described above, we had a pyridine binding site for precoordination of the ligand prior to the carbene metallation step. We wanted to see if a chelating bis-carbene could be formed in the absence of such prior binding. In collaboration with Eduardo Peris [27], we therefore looked at the imidazolium salts 7 with $\mathrm{Rh}$ and Ir precursors. As can be seen in eq. 7, the reaction proved successful in both cases to give $\left[\mathrm{M}\left(\mathrm{R}_{2}-\mathrm{MBI}\right) \mathrm{I}_{2}(\mathrm{OAc})\right]\left[\mathrm{R}_{2}-\mathrm{MBI}=N, N^{\prime}\right.$-dialkyl(methylene-bis-imidazolyl)]. An orthophenylene linker was equally successful to give $\left[\mathrm{M}\left(\mathrm{R}_{2}-\mathrm{BBI}\right) \mathrm{I}_{2}(\mathrm{OAc})\right]\left[\mathrm{R}_{2}-\mathrm{BBI}=N, N^{\prime}\right.$-dialkyl(benzene1,2-bis-imidazolyl)].

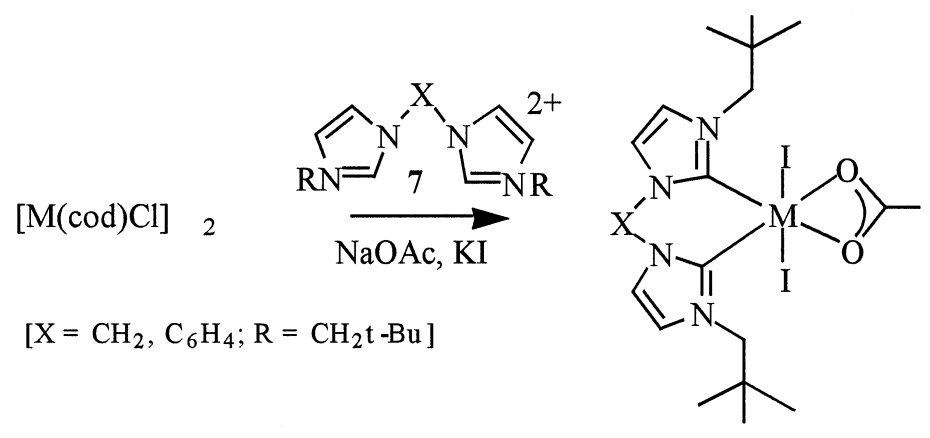

These compounds proved to be catalytically active for transfer hydrogenation of ketones using $i$-PrOH as reductant and solvent. Iridium proved to be more active than $\mathrm{Rh}$, and neopentyl ( $\mathrm{Np}$ ) was by far the best $\mathrm{R}$ group; this may be because it resists both Hoffmann degradation, lacking $\beta$-Hs and $\mathrm{R}$ group abstraction by $\mathrm{OH}^{-}$, because of the steric hindrance of the Np group. The great advantage of the catalyst is that it is perfectly indifferent to the presence of air and does not need purification of materials, unlike the classical phosphine catalysts. 


\section{Deactivation modes}

The catalysts discussed above certainly do deactivate, but the pathways are yet to be determined. Loss of carbene is not expected because the free carbene is so unstable relative to the bound form, but loss of imidazolium salt is likely to be thermodynamically and kinetically much more feasible. Many catalytic reactions involve intermediates containing $\mathrm{M}-\mathrm{H}$ or $\mathrm{M}-\mathrm{R}$ bonds that could in principle give reductive elimination with the carbene to give the imidazolium salt; substrate alkenes could also possibly insert into the $\mathrm{M}-\mathrm{C}$ bond of the carbene. Important prior work [13a] shows that reductive elimination is possible when a carbene is cis to an $\mathrm{M}-\mathrm{H}$ or $\mathrm{M}-\mathrm{R}$ bond and capable of achieving a conformation in which the $\mathrm{M}-\mathrm{X}$ bond is normal to the imidazole ring. Although this deactivation pathway is a potential Achilles heel for NHCs in catalytic applications, many catalysts operate perfectly successfully without significant deactivation. As always, catalysis is a kinetic phenomenon: If productive pathways are sufficiently rapid relative to deactivation, catalysis can be efficient. This model nevertheless implies that, although deactivation can probably never be stopped entirely, one should still look for ways to slow it relative to productive pathways. One way of achieving this may be incorporation of the NHC into chelates and pincers, and may favor cases like the twisted pincers discussed above where the NHC imidazole rings are not normal to any of the remaining ligand binding sites. Base conditions, such as in the hydrogen transfer reactions, place additional demands on the ligands, and further work is needed to improve ligand robustness.

\section{CONCLUSIONS AND FUTURE PROSPECTS}

These imidazolium-based carbenes are attractive because they bind metals very strongly as spectator ligands, give air and moisture stable complexes, and promote catalytic activity in a variety of situations. The starting imidazolium salts can readily be synthesized but in binding to a metal have a more complex chemistry than originally anticipated and in some cases form abnormal carbenes. They are therefore more difficult than phosphines to reliably attach to the metal, and it will be necessary to verify the structures of catalysts formed in situ from metal precursor and imidazolium salt. It should also be possible in the future to incorporate these ligands into complex architectures to allow application in supramolecular or sensor applications. Main group elements should be equally susceptible to binding, and the resulting organometallic species could give useful applications.

Combined theoretical/experimental work has been extremely useful in this area. Notably, the effect of the counterion on the formation of right vs. wrong carbene isomers was one result of this approach. Counterion effects may be more pervasive than currently believed.

\section{ACKNOWLEDGMENTS}

I thank Eduardo Peris (Castellòn), Odile Eisenstein and Eric Clot (Montpellier) and Jack Faller (Yale), the coworkers cited, and the DOE and Johnson-Matthey company for support.

\section{REFERENCES}

1. E. O. Fischer and A. Maasbol. Angew. Chem., Int. Ed. Engl. 3, 580 (1964).

2 (a) K. Öfele. J. Organomet. Chem. 12, 42 (1968); (b) H.-W. Wanzlick and H.-J.Schönherr. Angew. Chem. Int., Ed. Engl. 7, 141 (1968).

3. (a) W. A. Herrmann. Angew. Chem., Int. Ed. Engl. 41, 1290 (2002); (b) A. A. D. Tulloch, A. A. Danopoulos, G. J. Tizzard, S. J. Coles, M. B. Hursthouse, R. S. Hay-Motherwell, W. B. Motherwell. Chem. Commun. 1270 (2001); (c) R. H. Grubbs. Chimia 56, 22 (2002); (d) T. Ruther, M. C. Done, K. J. Cavell, E. J. Peacock, B. W. Skelton, A. H. White. Organometallics 20, 5522 (2001); (e) C. L. Yang and S. P. Nolan. Synlett 1539 (2001). 
4. C. A. Tolman. J. Am. Chem. Soc. 92, 2953 (1970); Chem. Rev. 77, 313 (1977).

5. G. Gelbard, H. B. Kagan, P. R. Stern. Tetrahedron 32, 233 (1976).

6 R. Noyori and H. Takaya. Acc. Chem. Res. 23, 345 (1990).

7. D. Bourissou, O. Guerret, F. P. Gabbai, G. Bertrand. Chem. Rev. 100, 39 (2000).

8. A. J. Arduengo, R. L. Harlow, M. Kline. J. Am. Chem. Soc. 113, 361 (1991).

9. D. H. Lee, J. Y. Chen, J. W. Faller, R. H. Crabtree. Chem. Commun. 213 (2001).

10. J. N. Coalter, G. Ferrando, K. G. Caulton. New J. Chem. 24, 835 (2000).

11. J. A. Loch, M. Albrecht, E. Peris, J. Mata, J. W. Faller, R. H. Crabtree. Organometallics 21, 700 (2002); E. Peris, J. A. Loch, J. Mata, R. H. Crabtree. Chem. Commun. 201 (2001).

12. (a) S. Gründemann, M. Albrecht, J. A. Loch, J. W. Faller, R. H. Crabtree. Organometallics 20, 5485 (2001); (b) O. Eisenstein, M. Albrecht, E. Clot, R. H. Crabtree, J. W. Faller, S. Grundemann, C. Megret, J. Miecznikowski. J. Chem. Soc., Dalton Trans. (2003). In press.

13. (a) D. S. McGuinness, K. J. Cavell, B. F. Yates, B. W. Skelton, A. H. White. J. Am. Chem. Soc. 123, 8317 (2001); (b) M. S. Viciu, G. A. Grasa, S. P. Nolan. Organometallics 20, 3607 (2001).

14. S. Gründemann, M. Albrecht, A. Kovacevic, J. W. Faller, R. H. Crabtree. J. Chem. Soc., Dalton Trans. 2163 (2002).

15. S. Gründemann, A. Kovacevic, M. Albrecht, J. W. Faller, R. H. Crabtree. Chem. Commun. 2274 (2001)

16. E. Peris, J. C. Lee, J. R. Rambo, O. Eisenstein, R. H. Crabtree. J. Am. Chem. Soc. 117, 3485 (1995).

17. A. Kovacevic, S. Gründemann, J. R. Miecznikowski, E. Clot, O. Eisenstein, R. H. Crabtree. Chem. Commun. 2580 (2002).

18. N. Kuhn, M. Walker, M. Steimann. Z. Naturforsch. B 57, 248 (2002).

19. S. Gründemann, A. Kovacevic, M. Albrecht, J. W. Faller, R. H. Crabtree. J. Am. Chem. Soc. 124, 10473 (2002).

20. (a) A. Macchioni, C. Zuccaccia, E. Clot, K. Gruet, R. H. Crabtree. Organometallics 20, 2367 (2001); (b) A. Macchioni. Unpublished data.

21. R. H. Crabtree, lecture I.234, ICCC34, Edinburgh, Scotland (2000).

22. R. J. Sundberg, R. F. Bryan, I. F. Taylor, H. Taube. J. Am. Chem. Soc. 96, 381 (1974).

23. K. L. Tan, R. G. Bergman, J. A. Ellman. J. Am. Chem. Soc. 124, 3202 (2002).

24. G. Sini, O. Eisenstein, R. H. Crabtree. Inorg. Chem. 41, 602 (2002).

25. L. Perrin, E. Clot, O. Eisenstein, J. Loch, R. H. Crabtree. Inorg. Chem. 40, 5806 (2001).

26. A. B. P. Lever. Inorg. Chem. 29, 1271 (1990).

27. M. Albrecht, R. H. Crabtree, J. Mata, E. Peris. Chem. Commun. 32, (2002) and unpublished data. 